\title{
Social Stress Scale
}

National Cancer Institute

\section{Source}

National Cancer Institute. Social Stress Scale. NCI Thesaurus. Code C121275.

A rating scale included in the Behavior Assessment System for Children that measures

the subject's feelings of stress and tension, and feeling excluded in personal relationships. 